\title{
Self-bending and asymmetric spatial self-phase modulation effects in nematic liquid-crystal films
}

\author{
Ru-Pin Pan \\ Department of Electrophysics, National Chiao Tung University, Hsinchu, Taiwan 30050, China \\ Shyu-Mou Chen and Ci-Ling Pan \\ Institute of Electro-Optical Engineering, National Chiao Tung University, Hsinchu, Taiwan 30050, China
}

Received July 18, 1990; accepted December 22, 1990

\begin{abstract}
Laser-induced beam bending and the far-field asymmetric diffraction ring pattern due to the self-bending and asymmetric spatial self-phase modulation (SPM) effects in nematic liquid-crystal films are investigated. The measured far-field patterns are in good agreement with numerical calculations based on the diffraction theory, taking into account the two dimensionality of the problem, the nonlocal effect, and the wave-front curvature effect. The individual contributions of the aforementioned effects to the far-field pattern are analyzed for the first time. It is shown that the outermost diffraction peak of the far-field pattern is not the strongest one and that a large fraction of the transmitted intensity remains in the central region (within $\pm 6 \mathrm{mrad}$ of the beam axis). Merging of some of the diffraction peaks in this region should also occur. Although the transmitted beam does deflect, the deflection angle does not increase monotonically with the incident laser intensity, as is the case for a purely Kerr-like medium. In the experiments discussed here, higher-order effects are present. As an example, it is shown that equally pronounced third- and fifth-order nonlinear optical SPM effects are responsible for an observed five-ring asymmetric ring pattern.
\end{abstract}

\section{INTRODUCTION}

The interaction of nonlinear-optical materials with a laser beam with an asymmetric intensity profile was first considered by Kaplan ${ }^{1}$ two decades ago. Exiting the medium, the beam was predicted to deflect from the original direction of propagation. This was referred to as the selfbending effect. In general, diffraction and self-focusing effects must also be considered. Self-bending is potentially useful in a number of applications, e.g., beam scanning, ${ }^{1}$ optical bistable devices, ${ }^{2}$ power limiting, ${ }^{3,4}$ measurement of the nonlinear index of refraction, ${ }^{4-7}$ and diagnostics of ultrafast processes. ${ }^{5,6,8}$ If the nonlinear film were thin enough, only the spatial phase profile of the transmitted beam would be altered. In this limit theoretical predictions of the far-field diffraction pattern due to a beam of light with a wedge $e^{4,9}$ or a semi-Gaussian profile ${ }^{9}$ in one dimension and a uniform profile in the other dimension were reported. It was also assumed in these studies that the third-order nonlinear effect is dominant. That is, the nonlinear index of refraction is linearly proportional to the laser intensity. Since the problem has been reduced to one dimension by these authors, it is not surprising that the predicted far-field pattern is identical to the power spectrum due to self-phase modulation (SPM) in the time domain ${ }^{10}:$ a semiperiodic structure of peaks and valleys, with the farthest peak from the beam axis being the strongest. The deflection angle of the beam, as defined by the angular position of the strongest peak, was predicted to be linearly proportional to the laser intensity. Earlier experimental studies included observations of the deflections of beams of a pulsed ruby laser in $\mathrm{NaCl}^{11}$ and semiconductor crystals ${ }^{12}$ and of a pulsed $\mathrm{CO}_{2}$ laser in $\mathrm{CS}_{2}$. ${ }^{5,6}$ In these experiments the nonlinear or optical-

0740-3224/91/051065-07\$05.00 field-induced phases $\phi_{\mathrm{NL}}$ were much less than $\pi$ rad. Self-focusing effects were also, in general, present. Recently, Swartzlander et al. ${ }^{13,14}$ reported experimental results on the self-deflection of a cw laser beam in sodium vapor near resonance. Self-bending angles as large as eight diffraction widths ( $5.9 \mathrm{mrad})$, as well as strong attenuation of the on-axis radiation, were observed. At $\simeq 200^{\circ} \mathrm{C}$ the self-deflection angle was found to increase linearly with laser power. That is, the nonlinear index of refraction was predominantly Kerr-like, or $\delta n=n_{2} I$, where $I$ is the laser intensity. Moderate saturation of the self-bending effect was also observed.

In this paper we present, to our knowledge for the first time, detailed measurements of the far-field diffraction pattern (up to 20 rings) exhibited by a normally incident asymmetric cw laser beam traversing a nematic liquidcrystal (NLC) film. NLC films were selected because of their large nonlinearity. ${ }^{15}$ Thin films can thus be used such that the self-focusing effect and the diffraction effect in the film itself are negligible. Previously, Khoo et al. ${ }^{16}$ studied optical limiting by the self-bending effect in a NLC film. A low-power inclined-incident $\mathrm{cw} \mathrm{Ar}^{+}$ion laser beam was used. Rings in the far-field pattern were also reported and were attributed to the spatial SPM effect ${ }^{17}$ for the asymmetric case. The deflection angle of the beam was found to be close to that of theoretical predictions of a Kerr-like medium. In a preliminary report we showed that equally pronounced third- and fifth-order SPM effects are simultaneously present in a five-ring asymmetric diffraction ring pattern. ${ }^{18}$ Further experiments permitted quantitative comparisons of experimental results with numerical calculations based on the diffraction theory, taking into account the two dimen- 


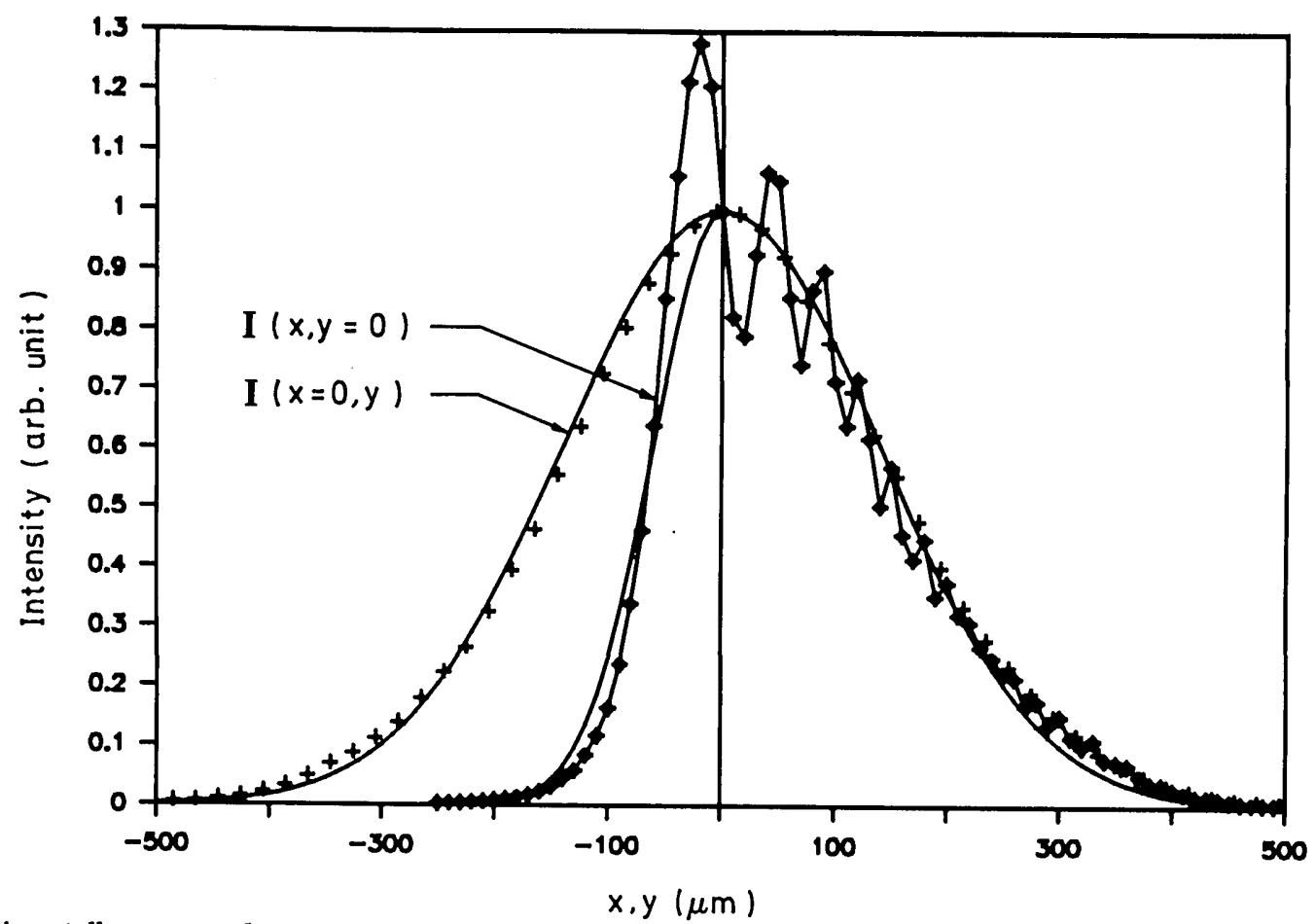

Fig. 1. Experimentally measured transverse profile of the laser beam at the sample position. In the $y$ direction, $I(x=0, y) \propto$ $\exp \left(-y^{2} / 2 \sigma_{I y}{ }^{2}\right)$, where $\sigma_{I y}=140 \mu \mathrm{m}$; the crosses are the experimental data points. In the $x$ direction, $I(x, y=0)$ was fitted with two semi-Gaussian functions with $\sigma_{I x}=60 \mu \mathrm{m}$ for $x<0$ and $\sigma_{I x}=140 \mu \mathrm{m}$ for $x>0$; the diamonds are the experimental data points.

sionality of the problem, the nonlocal effects, ${ }^{19,20}$ and the wave-front curvature effect. ${ }^{21,22}$ The contributions of the aforementioned effects to the far-field pattern will be discussed. In addition, we will show the evolution of laser beam bending and other features in the central region (within \pm 6 mrad of the beam axis) of the far-field pattern as the laser intensity changes.

\section{EXPERIMENTAL METHODS}

Our experimental setup was typical. ${ }^{16,17} \mathrm{~A} \mathrm{cw} \mathrm{Ar}{ }^{+}$laser beam was focused by a lens and partially blocked $(\approx 25 \%)$ by a knife edge approximately $1.5 \mathrm{~cm}$ in front of a homeotropically aligned NLC cell of 4-cyano- $4^{\prime}$-pentybiphenyl (5CB) at $25.0 \pm 0.1^{\circ} \mathrm{C}$. The thickness of the cell was $350 \mu \mathrm{m}$. The laser beam was normally incident on the sample with an asymmetric spatial profile as shown in Fig. 1. We measured the beam profile by translating a pinhole (10- $\mu \mathrm{m}$ diameter) across the beam and recording the transmitted power. Minor undulations were due to diffraction of the knife edge. Their effects on our results will be discussed in Section 3. The transverse profile of the laser beam along the $x$ direction could be approximated by two halves of semi-Gaussian profiles with $e^{-2}$ diameters of $\sigma_{I x}=60 \mu \mathrm{m}$ for $x<0$ and $\sigma_{I x}=140 \mu \mathrm{m}$ for $x>0$, where the $x$ axis is the dimension with the transverse profile asymmetry. In the $y$ direction the laser intensity profile is a symmetric Gaussian function with an $e^{-2}$ diameter of $\sigma_{I y}=140 \mu \mathrm{m}$. The $z$ axis is along the propagation direction of the laser. The sample was placed at a position in front of the focus of a positive lens ( $f=$ $75 \mathrm{~cm}$ ) in the convergent beam. Beam diameters were measured at the sample and at another position between the lens and the sample. Since the distance between these two positions was also known, using similar trian- gles we estimated the radius of curvature of the beam at the sample to be $R \cong-25 \mathrm{~cm}$. We used a charge-coupled device camera with $512 \times 512$ pixels to record the observed diffraction pattern on a screen approximately $350 \mathrm{~cm}$ away from the sample. The laser intensity was adjusted such that either a 5-, 10-, or 20-ring pattern appeared on the screen. To accomplish this, we used a variable attenuator consisting of a Glan laser polarizer and a half-wave plate. With the sample out of the beam, we did not observe deviation of the beam for different settings of the half-wave plate.

\section{THEORETICAL METHODS}

We calculated the theoretical far-field pattern for an asymmetric Gaussian beam propagating through a thin NLC film of thickness $d$ with the use of the Hankel-KirchhoffFraunhofer integral, taking into account the two dimensionality of the problem, the linear diffraction effect, the wave-front curvature effect, and the nonlocal effect ${ }^{15,21,22}$ :

$$
\begin{aligned}
I(\alpha) \propto & \mid \int_{-\infty}^{\infty} \cdot \int \mathrm{d} x \mathrm{~d} y E(x, y) \exp [-i k x \sin \alpha \\
& \left.+i \phi_{R}(x, y)+i \phi_{\mathrm{NL}}(x, y)\right]\left.\right|^{2},
\end{aligned}
$$

where $\alpha$ is the diffraction angle, $k=2 \pi / \lambda$, and $E(x, y) \propto$ $[I(x, y)]^{1 / 2} \propto \exp \left(-x^{2} / 4 \sigma_{I x}{ }^{2}\right) \exp \left(-y^{2} / 4 \sigma_{I y}{ }^{2}\right)$ is the optical field amplitude. An experimentally measured $E(x, y)$ (see Fig. 1) was also incorporated into relation (1). The effect of the undulations was found to be minimal. The asymmetric Gaussian profile is used for all the calculations performed in this paper. Also in relation (1), $\phi_{R}=$ $k\left(x^{2}+y^{2}\right) / 2 R$ is the optical phase shift induced by the wave-front curvature, and $\phi_{\mathrm{NL}}(x, y)=k \int_{0}^{d} \delta n(x, y, z) \mathrm{d} z$ is 
the nonlinear phase induced by the optical-field-induced index of refraction $\delta n$. In most nonlinear-optical materials, $\delta n \propto E^{2}$ if the lowest order of nonlinearity is the third order. As a result, $\phi_{\mathrm{NL}}$ should have the same Gaussian intensity profile of the laser beam. In a NLC film, however, the situation is more complicated, since $\delta n$ is related to the molecular reorientation angle $\theta$ by

$$
\delta n(x, y, z)=\left(n_{o}^{-2} \cos ^{2} \theta+n_{e}^{-2} \sin ^{2} \theta\right)^{-1 / 2}-n_{o},
$$

where $n_{0}$ and $n_{e}$ are the ordinary and extraordinary indices of refraction of the NLC film. For samples with hard boundaries and a laser intensity not far above the Freedericksz transition, $\theta(x, y, z) \cong \theta_{0} \theta_{x}(x) \theta_{y}(y)$ $\sin [(\pi / d) z]$, and this can be calculated from the equation of the torque balance. Here $\theta_{0}$ is the maximum reorientation angle; $\theta_{x}(x)$ and $\theta_{y}(y)$ denote the functional dependences in $x$ and $y$ of the reorientation angle $\theta(x, y, z)$. We assume that $\theta_{x}(x)$ for $x>0$ and $\theta_{y}(y)$ obey the same equation as that for the reorientation angle $\theta(r)$ in symmetric SPM with $\sigma_{I}=\sigma_{I x}=\sigma_{I y}=140 \mu \mathrm{m}$; that is, ${ }^{15}$

$$
\begin{array}{r}
\frac{\mathrm{d}^{2} \theta(r)}{\mathrm{d} r^{2}}+\frac{1}{r} \frac{\mathrm{d} \theta(r)}{\mathrm{d} r}+\left(\frac{n_{e}^{2}-n_{o}^{2}}{c n_{o} K}\right) I(r)\left[\theta(r)-\frac{1}{2} \theta^{3}(r)\right] \\
-\left(\frac{\pi}{d}\right)^{2} \theta(r)=0,
\end{array}
$$

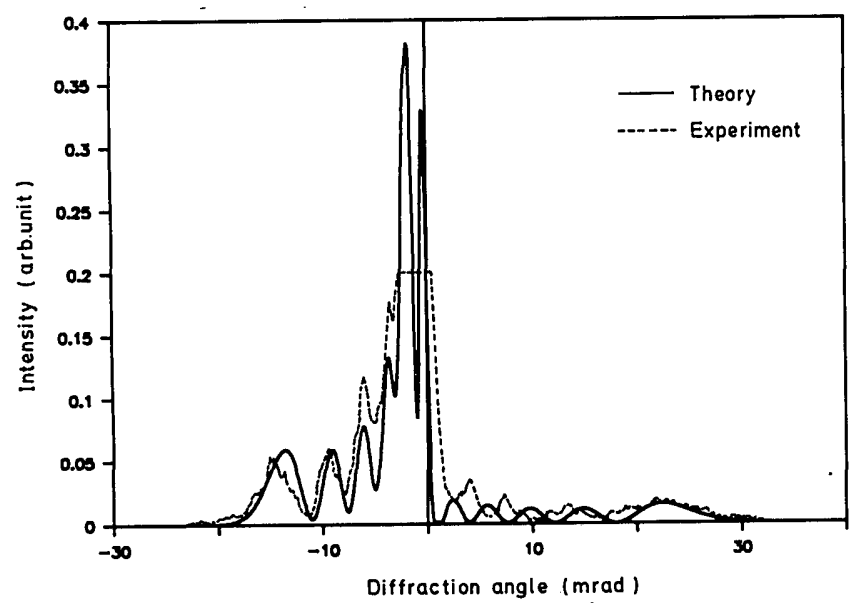

(a)

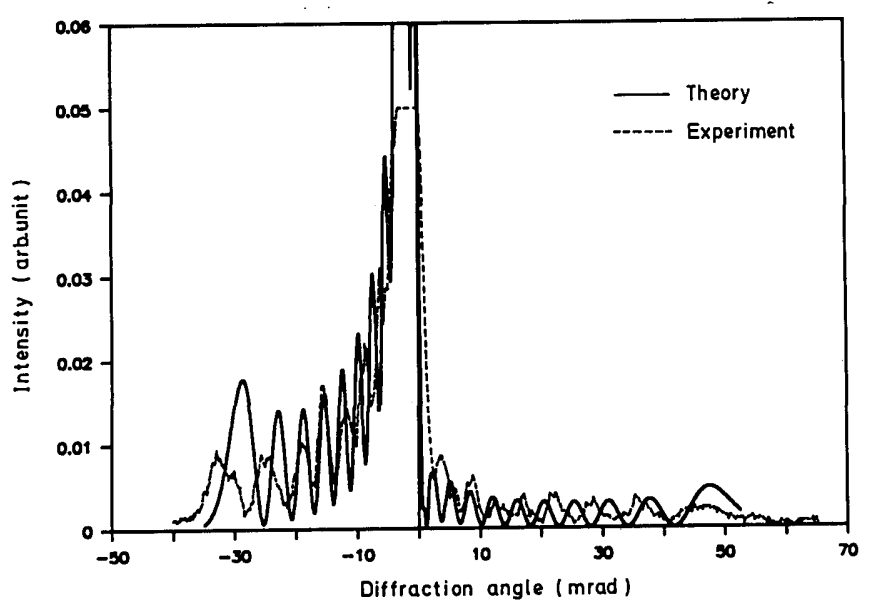

(b) where $I(r)=I_{0} \exp \left(-r^{2} / 2{\sigma_{I}}^{2}\right)$ and $K$ is the elastic constant of the NLC in the one-constant approximation. In NLC films the reorientation motions of the molecules are strongly correlated. The response of the molecules at a certain point depends on the reorientation motion of the molecules elsewhere. This is the well-known nonlocal effect. Because of this effect the molecules do not respond to sharp changes in the transverse profile of the light beam. It was found that the undulations shown in Fig. 1 can be ignored in the evaluation of the reorientation angle. Similarly we could calculate $\theta_{x}(x)$ for $x<0$ with $\sigma_{I x}=$ $\sigma_{I y}=\sigma_{I}=60 \mu \mathrm{m}$. We numerically solved Eq. (3) by following standard Runge-Kutta procedures ${ }^{23}$ with appropriate boundary conditions. With $n_{o}=1.54, n_{e}=1.73$, $K=0.82 \times 10^{-6} \mathrm{dyn}$, and $I_{0}$ corresponding to diffraction ring patterns with a certain number of rings, we calculated the transverse dependence of $\theta$ and $\phi_{\mathrm{NL}}$. We then substituted these into relation (1) to obtain the far-field pattern of the transmitted beam.

\section{RESULTS AND DISCUSSION}

The theoretical diffraction patterns for $N$ rings $(N=5$, 10, and 20) predicted by relation (1) are in good agreement with the experimental results (see Fig. 2), in light of the approximations that were taken. The central region of

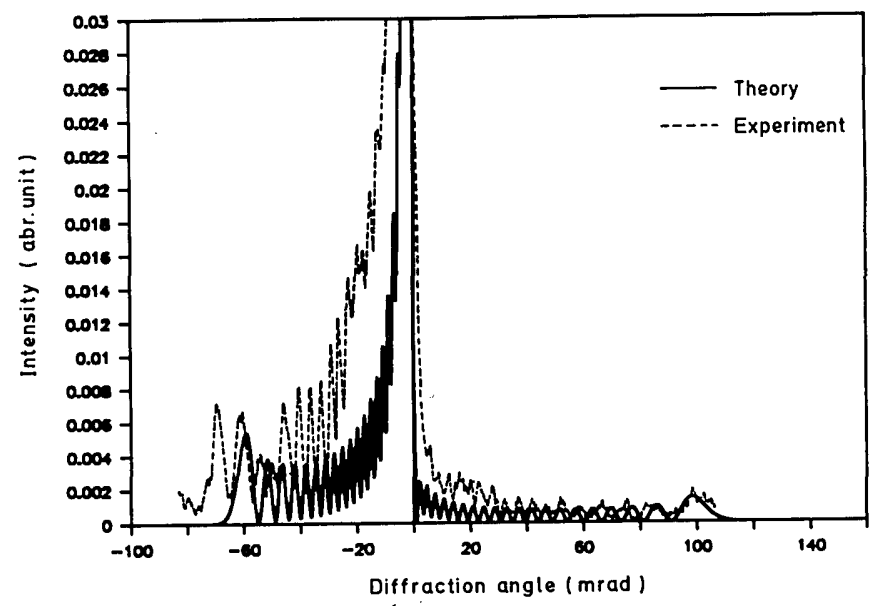

(c)
Fig. 2. Experimental and theoretical far-field patterns at $y=0$ for a laser beam normally incident upon a homeotropically aligned $5 \mathrm{CB}$ nematic cell of $350-\mu \mathrm{m}$ thickness, generating (a) 5 , (b) 10 , and (c) 20 rings. 


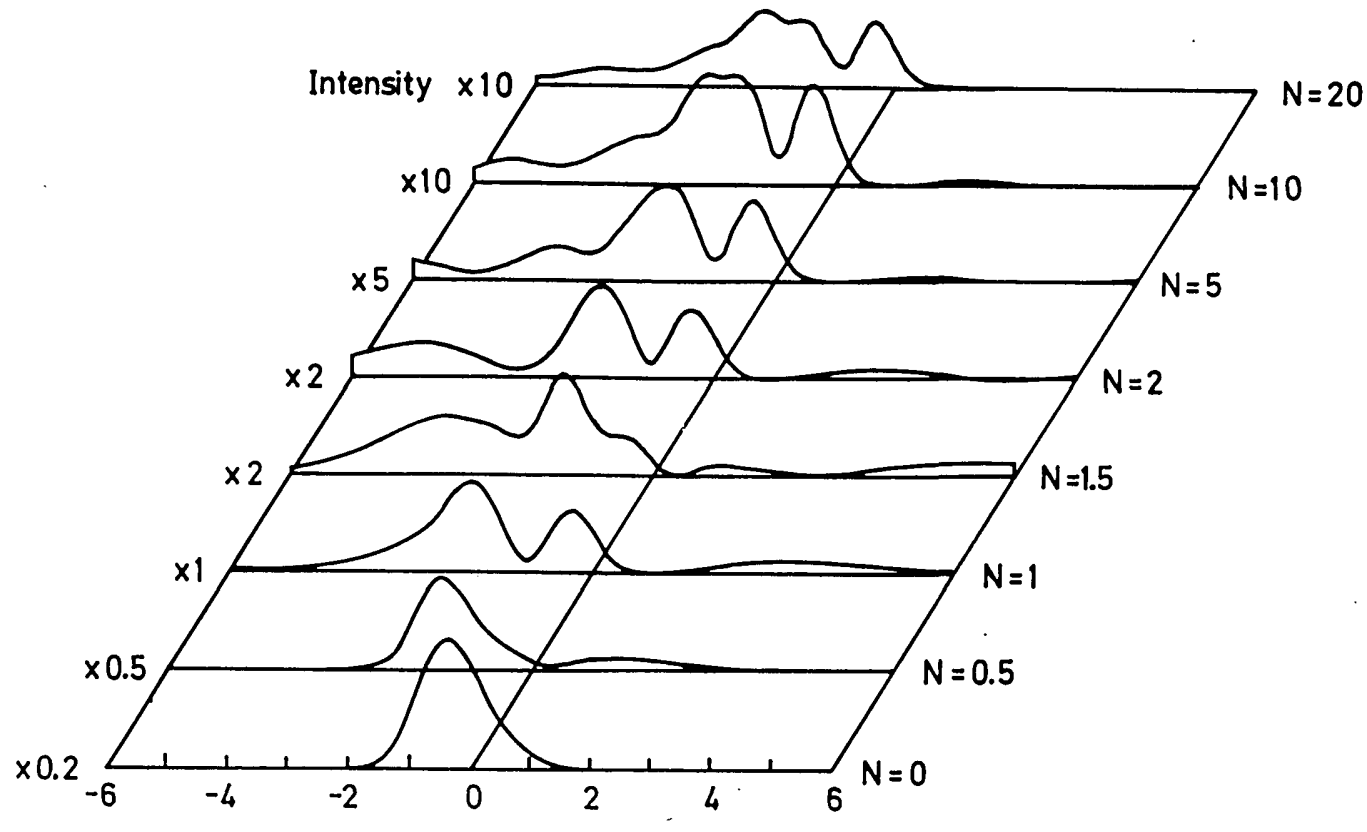

\section{Diffraction angle (mrad)}

Fig. 3. Detailed features of the central region of the far-field pattern ( \pm 6 mrad from the $z$ axis) with ring numbers $N=0,0.5,1,1.5,2,5$, 10, and 20. The actual transmitted laser intensity should be divided by the scale factors shown here.

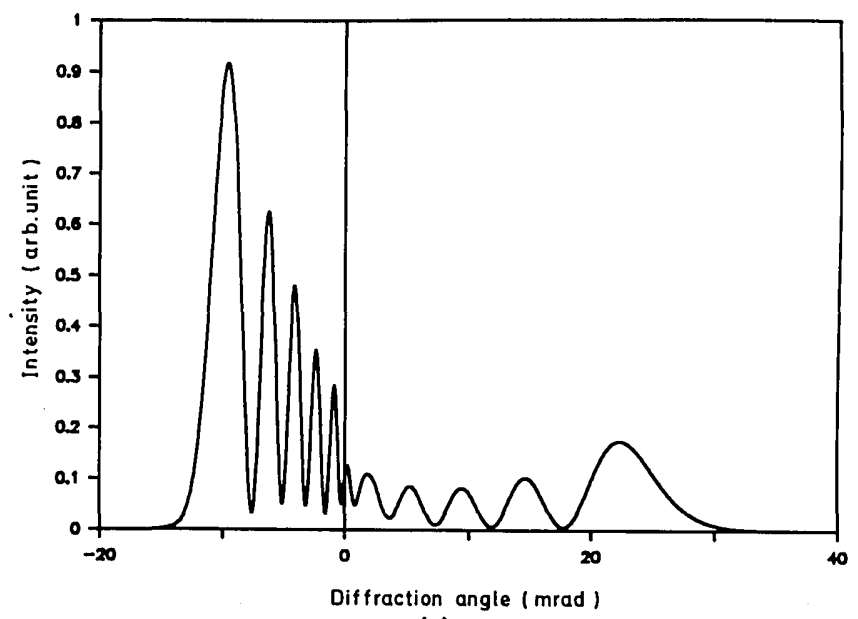

(a)

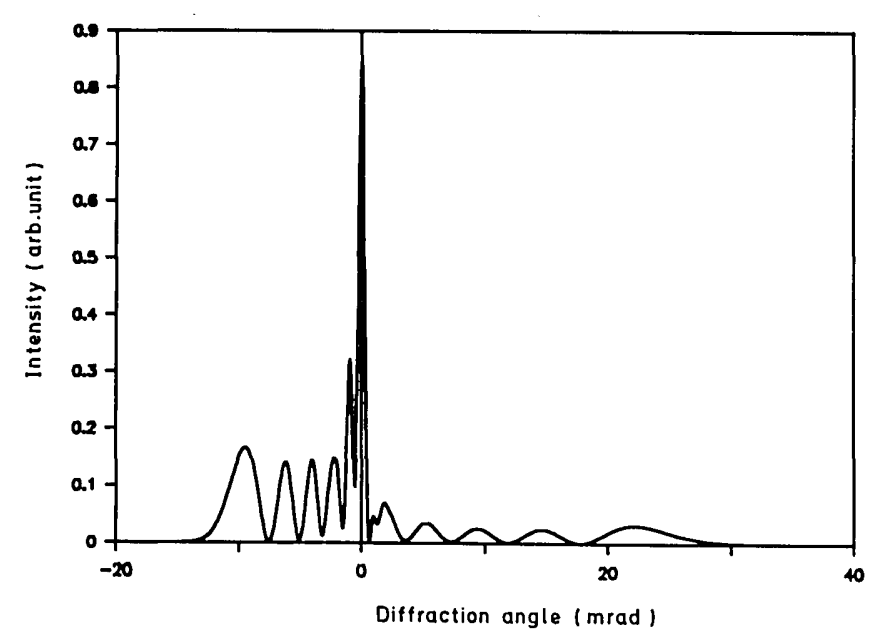

(b)

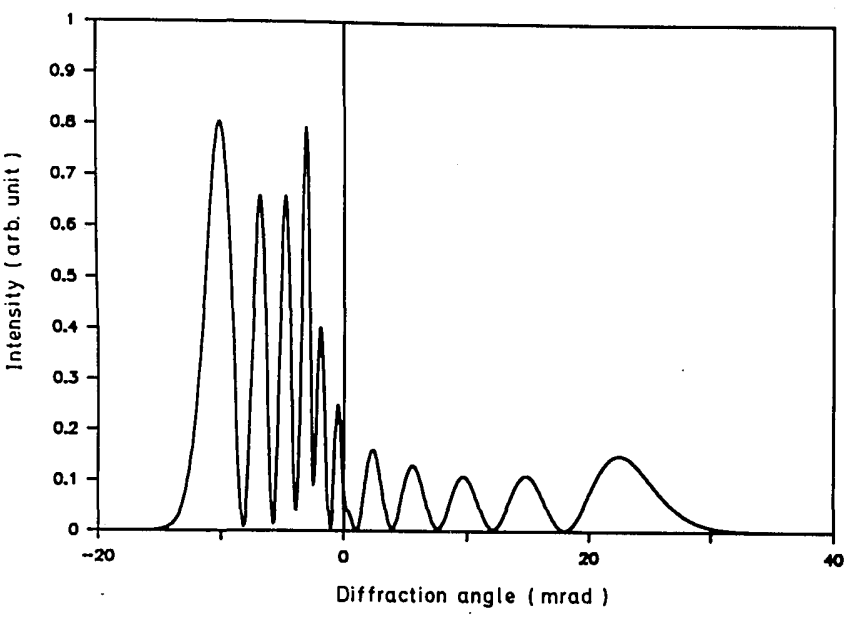

(c)

Fig. 4. Asymmetric five-ring diffraction pattern predicted by relation (1): (a) only the one-dimensional case is treated, the wave-front curvature and nonlocal effects being neglected; (b) the two dimensionality of the problem is taken into account, the wave-front curvature and the nonlocal effects still being neglected; (c) same as (b), except that the wave-front curvature effect is considered. 
the observed rings was not resolved because of saturation of the charge-coupled device camera used. Nevertheless, it is apparent that a large fraction of the transmitted intensity remains in the central region of the far-field pattern (within $\pm 6 \mathrm{mrad}$ of the beam axis). In addition, the outermost diffraction peak is not the strongest peak, in contrast with the case of a purely Kerr-like medium. Similar features were discussed briefly by previous authors investigating the symmetric spatial SPM effects in NLC films. ${ }^{22,24-26}$ However, there were few reports directly comparing the observed symmetric far-field diffraction pattern with the theory. ${ }^{21,24}$ A satisfactory fit was found for laser intensities generating up to two rings in a thin hybrid nematic cell and negligible nonlocal and wavefront curvature effects. ${ }^{24}$ An anomalously high intensity of the central peak was attributed to a temperaturedependent index of refraction..$^{24,26}$ In our study the highest incident laser intensity used was less than $170 \mathrm{~W} / \mathrm{cm}^{2}$. The liquid-crystalline material investigated, 5CB, is nearly transparent at the laser wavelength $(\lambda=$ $514.5 \mathrm{~nm}$ ). The laser heating effect should be negligible. We cannot rule out thermally induced saturation of the self-deflection effect, however, because of saturation of the charge-coupled device camera used.

We show in Fig. 3 detailed features of the central region for asymmetric ring patterns with ring numbers $N=0$, $0.5,1,1.5,2,5,10$, and 20 as predicted by numerical calculations based on the formulas outlined in Section 3. Examining Figs. 2 and 3, we conclude that the laser beam does bend in the sense that the position of peak laser intensity shifts. However, the amount of bending is relatively small. At the highest laser intensity studied, generating 20 rings, the maximum amount of beam bending is predicted to be $2.2 \mathrm{mrad}$. This corresponds to a shift of the beam axis of the incident laser by approximately one beam diameter. For the larger ring numbers, e.g., $N=$ 10 and 20 , merging of some of the diffraction peaks in the central region is also predicted. We note also that the deflection angle does not increase monotonically with the number of rings. The features in the central region depend critically on the wave-front curvature effect. ${ }^{21,22}$ By changing $R$ from negative to positive values, we can alter drastically the intensity distribution. ${ }^{22}$

At this point it is useful to examine the effects of various terms on the right-hand side of relation (1). The asymmetric five-ring pattern is used as an example. In the first case we assume that $\phi_{\mathrm{NL}} \propto I$, i.e., $\sigma_{\phi}=\sigma_{I}$, instead of using Eqs. (2) and (3) in the calculation of $\phi_{\mathrm{NL}}(x, y)$; we treat the beam propagation problem as one dimensional; and we neglect the wave-front curvature effect as well. The far-field pattern predicted by relation (1) is shown in Fig. 4(a). As one might expect, this is identical to the time-domain results and to those of Ref. 9. In this case the diffraction angle of the outermost peak, which is also the strongest peak, is linearly proportional to the incident laser intensity. Taking into account the two dimensionality of the problem, we illustrate the far-field pattern in Fig. 4(b). A pronounced central peak emerges. With the wave-front curvature effect added, the far-field pattern evolved into that shown in Fig. 4(c). The final result of taking into consideration all the effects mentioned above and the nonlocal effect was already shown in Fig. 2 above.

Since the diffraction angle of the outermost ring is $\left|\alpha_{x}\right| \propto|\mathrm{d} \phi(x, y) / \mathrm{d} x|_{\max } \propto 1 / \sigma_{\phi x}$, we expect the asymmetry ratio of the diffraction pattern to be $\left|\alpha_{x>0}\right|_{\max } /\left|\alpha_{x<0}\right|_{\max } \cong$ $\sigma_{\phi, x<0} / \sigma_{\phi, x<0}$. For an asymmetric five-ring pattern the theoretical asymmetric ratio is 1.6. This is in excellent agreement with our experimental result, which is 1.5. In contrast, the value of this ratio would be 2.35 if $\phi_{\mathrm{NL}}$ had exhibited a Gaussian profile with the same width as the

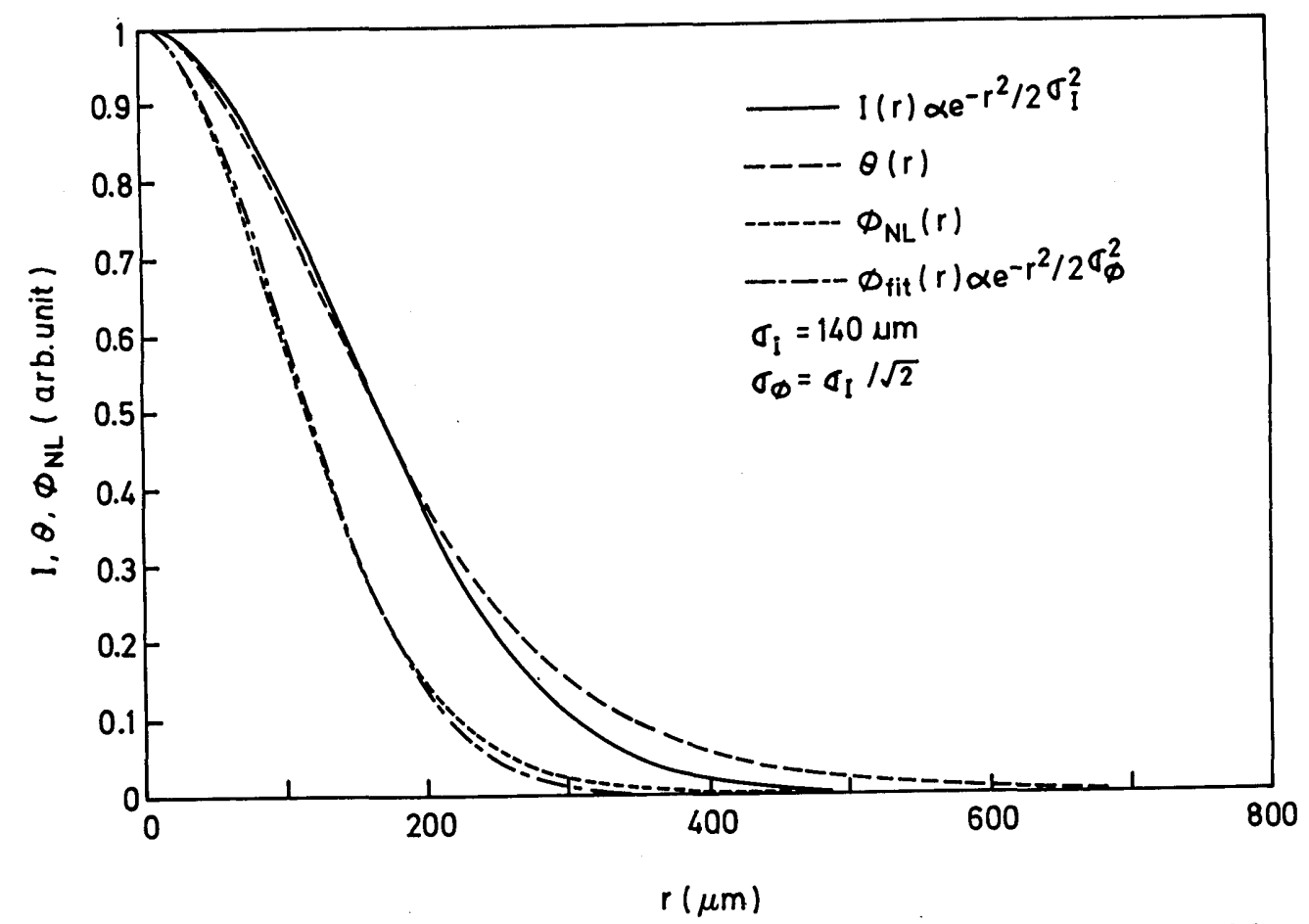

Fig. 5. Transverse profile of the reorientation angle $\theta$ and the optical-field-induced phase shift $\phi_{\mathrm{NL}}$ as a function of the transverse dimenFig. 5. Transverse profile of the reorientation angle $\theta$ and the optical-field-induced phase shift $\phi_{\mathrm{NL}}$ as a function
sion $r$ for a symmetric Gaussian intensity profile with $\sigma_{I}=140 \mu \mathrm{m} . \quad \phi_{\text {fit }}$ is a Gaussian function with $\sigma_{\phi}=\sigma_{I} / \sqrt{2}$. 


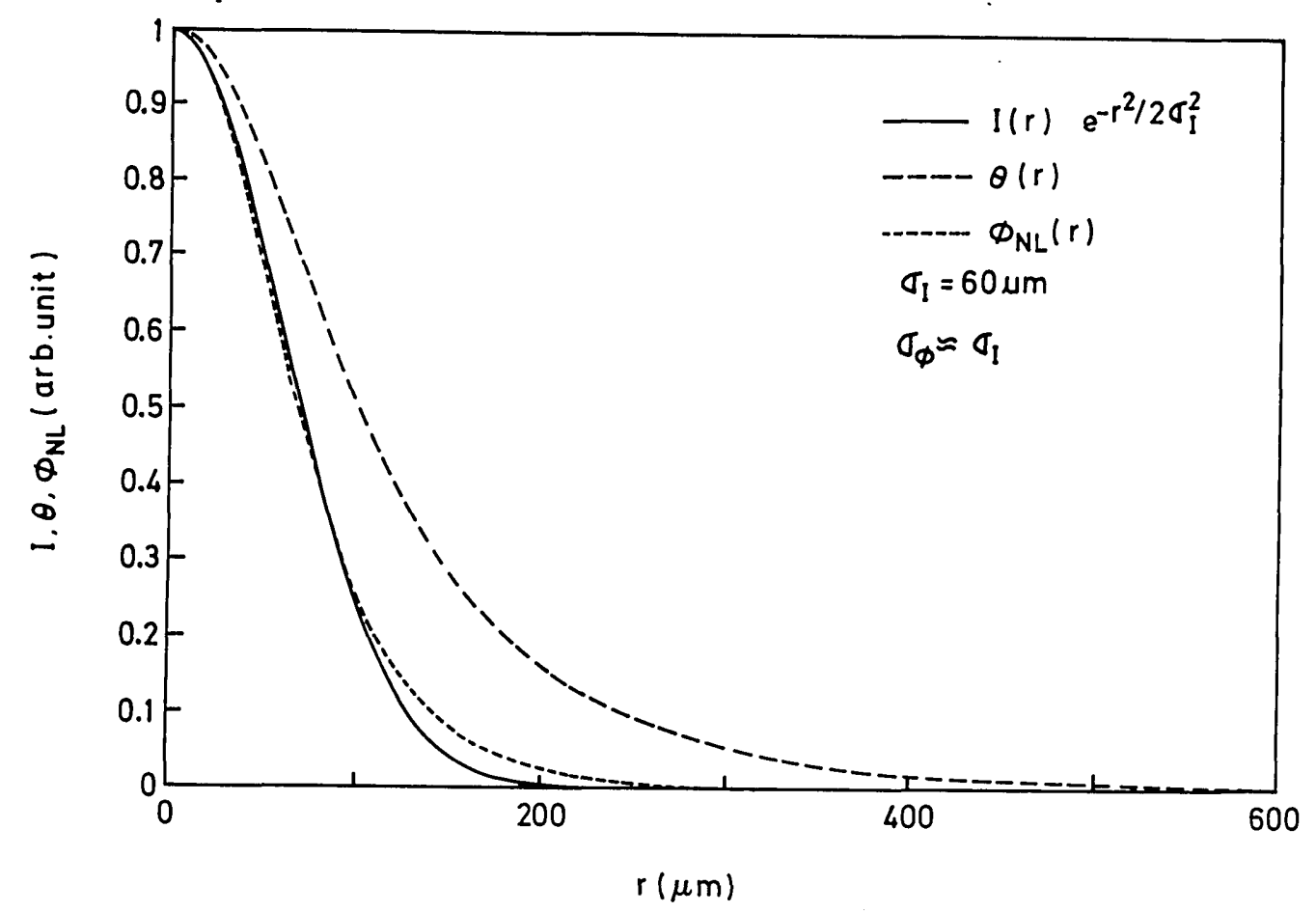

Fig. 6. Transverse profile of the reorientation angle $\theta$ and the optical-field-induced phase shift $\phi_{\mathrm{NL}}$ as a function of the transverse dimension $r$ for a symmetric Gaussian intensity profile with $\sigma_{I}=60 \mu \mathrm{m}$. $\phi_{\text {fit }}$ is a Gaussian function with $\sigma_{\phi} \approx \sigma_{I}$.

incident laser beam. The agreement is somewhat worse for larger ring-numbers but still within $15 \%$ of our theoretical predictions.

Because of its large nonlinearity the nonlinear index of refraction of a NLC film is in general a complex function of the incident laser intensity. In this sense many orders of the nonlinear index of refraction would contribute to $\phi_{\mathrm{NL}}$. This is primarily due to the so-called nonlocal effect mentioned above. As a result, $\phi_{\mathrm{NL}}$ does not exhibit the same profile of the incident laser beam. Only in the limit of small reorientation angles and for an inclined incident laser beam is $\phi_{\mathrm{NL}}$ linearly proportional to the laser intensity. In that case, which corresponds to that of Ref. 16, the spatial SPM phenomenon is essentially a third-order effect. In general, many orders of spatial SPM effects could have contributed to the observed ring patterns. In fact, it is possible to select experimental parameters, e.g., laser intensity, beam diameter, and sample thickness, such that the SPM effect of a particular order is dominant. As an example, the theoretical transverse dependences of $\theta$ and $\phi_{\mathrm{NL}}$ calculated from Eqs. (2) and (3) for a normally incident laser beam with a Gaussian intensity profile and $\sigma_{I x}=\sigma_{I y}=\sigma_{I}=140 \mu \mathrm{m}$, generating a fivering pattern in a $5 \mathrm{CB}$ film of $350-\mu \mathrm{m}$ thickness, are plotted as a function of the transverse dimension $r$ in Fig. 5. Clearly $\phi_{\mathrm{NL}}$ can be fitted by a Gaussian function with an $e^{-2}$ diameter of $\sigma_{\phi}=\sigma_{I} / \sqrt{2}$; that is, $\phi_{\mathrm{NL}} \propto I^{2} \propto E^{4}$. We interpret this as a manifestation of the fifth-order spatial SPM effect, in accordance with the usual definition for high-order optical-field-induced refractive index effects. Results for the case of $\sigma_{I}=60 \mu \mathrm{m}$ are shown in Fig. 6 . In contrast with the case of $\sigma_{I}=140 \mu \mathrm{m}, \phi_{\mathrm{NL}}$ can also be fitted here extremely well by a Gaussian profile, but with an $e^{-2}$ diameter of $\sigma_{\phi} \approx \sigma_{I}$, or $\phi_{\mathrm{NL}} \propto I$. This corresponds to a third-order SPM effect. Thus our experimental configuration for the observation of an asymmetric five-ring pattern corresponds to an extremely interesting situation in which the SPM effect in the positive- $x$ half-space is of the fifth order, while in the negative- $x$ half-space it is of the third order. Furthermore, the third- and fifth-order effects are equally pronounced. The fact that the theoretical diffraction ring pattern predicted by relation (1) agrees with the experimental results quite well (see Fig. 2 above) gives credence to our analysis.

\section{CONCLUSIONS}

We studied the nonlinear interaction of an asymmetric laser beam with NLC films both experimentally and theoretically. The spatial SPM effect was shown to be responsible for the asymmetric diffraction rings (up to 20) observed in the far field. In the thin-film approximation the so-called self-bending effect, manifested as a deflection of the whole beam, was shown to be but one attribute of the spatial SPM effect. Experimental results were found to be in good agreement with numerical calculations based on the diffraction theory, taking into account the two dimensionality of the problem, the wave-front curvature effect, and the nonlocal effect. The individual contributions of the aforementioned effects to the far-field pattern were analyzed for the first time. In contrast with the case of a purely Kerr-like medium, we showed that for NLC films under our experimental conditions the outermost diffraction peak was not the strongest peak and that a large fraction of the redistributed intensity remained in the central region (within $\pm 6 \mathrm{mrad}$ of the beam axis). While the beam did bend in the sense that the position of peak intensity shifted, the amount of bending was quite small and did not increase monotonically with the incident laser intensity. At the highest laser intensity studied, generating 20 rings, the maximum beam deflection angle was predicted to be only $2.2 \mathrm{mrad}$. For higher 
laser intensity incident on the NLC film, it was also predicted that some of the diffraction peaks would merge. Finally, we showed that experimental parameters could be tailored such that the spatial SPM effect of a particular order in a NLC film was dominant. As an example, equally pronounced third- and fifth-order effects were identified in an asymmetric five-ring diffraction pattern.

\section{ACKNOWLEDGMENTS}

This work was partially supported by the National Science Council of the Republic of China under grant NSC790208-M009-30. C.-L. Pan acknowledges helpful discussions with Chiao-Yao She of Colorado State University and Y. R. Shen of the University of California, Berkeley.

\section{REFERENCES}

1. A. E. Kaplan, JETP Lett. 9, 33 (1969).

2. A. E. Kaplan, Opt. Lett. 6, 360 (1981).

3. J. A. Hermann, Opt. Commun. 62, 367 (1987); Opt. Quantum Electron. 19, 169 (1987).

4. M. J. Aileau, W. E. Williams, and E. W. Van Stryland, IEEE J. Quantum Electron. QE-19, 731 (1983); J. A. Hermann, J. Opt. Soc. Am. B 1, 729 (1984).

5. I. Golub, Y. Beaudoin, and S. L. Chin, Opt. Lett. 13, 488 (1988).

6. I. Golub, Y. Beaudoin, and S. L. Chin, J. Opt. Soc. Am. B 5, 2490 (1988).

7. M. Sheik-bahae and E. W. Van Stryland, Opt. Lett. 14, 955 (1989).

8. P. B. Corkum and D. Keith, J. Opt. Soc. Am. B 2, 1873 (1985).
9. G. A. Swartzlander, Jr., and A. E. Kaplan, J. Opt. Soc. Am. B 5, 765 (1988).

10. See, for example, Y. R. Shen and Guo-Zhen Yang, in The Supercontinuum Laser Source, R. R. Alfano, ed. (SpringerVerlag, New York, 1989), Chap. 1, and references therein.

11. M. S. Brodin and A. M. Kamuz, JETP Lett. 9, 351 (1969).

12. A. A. Borshch, M. S. Brodin, V. I. Volkov, and V. V. Ovchar, Sov. J. Quantum Electron. 5, 340 (1975).

13. G. A. Swartzlander, Jr., H. Yin, and A. E. Kaplan, Opt. Lett. 13, 1011 (1988).

14. G. A. Swartzlander, Jr., H. Yin, and A. E. Kaplan, J. Opt. Soc. Am. B 6, 1317 (1989).

15. See, for example, I. C. Khoo, Prog. Opt. 26, 105 (1988), and references therein.

16. I. C. Khoo, G. M. Finn, R. R. Michael, and T. H. Liu, Opt. Lett. 11, 227 (1986).

17. S. D. Durbin, S. M. Arakelian, and Y. R. Shen, Opt. Lett. 9, 584 (1981).

18. S.-M. Chen, R.-P. Pan, and C.-L. Pan, Opt. Commun. 79, 469 (1990).

19. L. Csillag, I. Jánossy, V. F. Kitaeva, N. Kroo, and N. N. Sobolev, Mol. Cryst. Liq. Cryst. 84, 125 (1982).

20. I. C. Khoo, T. H. Liu, and R. Normandin, Mol. Cryst. Liq. Cryst. 131, 315 (1985).

21. E. Santamato and Y. R. Shen, Opt. Lett. 9, 584 (1984).

22. I. C. Khoo, J. Y. Hou, T. H. Liu, P. Y. Yan, R. R. Michael, and G. M. Finn, J. Opt. Soc. Am. B 4, 886 (1987).

23. See, for example, R. W. Hornbeck, Numerical Methods (Prentice-Hall, Englewood Cliffs, N.J., 1982), pp. 194-196.

24. F. Bloisi, L. Vicari, F. Simoni, G. Cipparrone, and C. Umeton, J. Opt. Soc. Am. B 5, 2462 (1988).

25. J. Eichler, R. Macdonald, and C. Dettmann, Mol. Cryst. Liq. Cryst. 174, 153 (1989).

26. F. Bloisi, L. Vicari, and F. Simoni, Opt. Commun. 53, 261 (1990). 